\title{
Results of an all-sky high-frequency Einstein@Home search for continuous gravitational waves in LIGO's fifth science run
}

\author{
Avneet Singh, ${ }^{1,2,3, *}$ Maria Alessandra Papa, ${ }^{1,2,4, \dagger}$ Heinz-Bernd Eggenstein, ${ }^{2,3}$ Sylvia Zhu, ${ }^{1,2}$ Holger Pletsch, ${ }^{2,3}$ \\ Bruce Allen, ${ }^{2,4,3}$ Oliver Bock, ${ }^{2,3}$ Bernd Maschenchalk, ${ }^{2,3}$ Reinhard Prix, ${ }^{2,3}$ and Xavier Siemens ${ }^{4}$ \\ ${ }^{1}$ Max-Planck-Institut für Gravitationsphysik, am Mühlenberg 1, 14476 Potsdam-Golm, Germany \\ ${ }^{2}$ Max-Planck-Institut für Gravitationsphysik, Callinstraße 38, 30167 Hannover, Germany \\ ${ }^{3}$ Leibniz, Universität Hannover, Welfengarten 1, 30167 Hannover, Germany \\ ${ }^{4}$ University of Wisconsin-Milwaukee, Milwaukee, Wisconsin 53201, USA
}

(Received 6 July 2016; published 26 September 2016)

\begin{abstract}
We present results of a high-frequency all-sky search for continuous gravitational waves from isolated compact objects in LIGO's fifth science run (S5) data, using the computing power of the Einstein@Home volunteer computing project. This is the only dedicated continuous gravitational wave search that probes this high-frequency range on S5 data. We find no significant candidate signal, so we set $90 \%$ confidence level upper limits on continuous gravitational wave strain amplitudes. At the lower end of the search frequency range, around $1250 \mathrm{~Hz}$, the most constraining upper limit is $5.0 \times 10^{-24}$, while at the higher end, around $1500 \mathrm{~Hz}$, it is $6.2 \times 10^{-24}$. Based on these upper limits, and assuming a fiducial value of the principal moment of inertia of $10^{38} \mathrm{~kg} \mathrm{~m}^{2}$, we can exclude objects with ellipticities higher than roughly $2.8 \times 10^{-7}$ within $100 \mathrm{pc}$ of Earth with rotation periods between 1.3 and 1.6 milliseconds.
\end{abstract}

DOI: 10.1103/PhysRevD.94.064061

\section{INTRODUCTION}

Ground-based gravitational wave (GW) detectors will be able to detect a continuous gravitational wave signal from a spinning deformed compact object provided that it is spinning with a rotational period between roughly 1 and 100 milliseconds, that it is sufficiently close to Earth and sufficiently "bumpy". Blind searches for continuous gravitational waves probe the whole sky and broad frequency ranges, looking for this type of objects.

In this paper, we present the results of an all-sky Einstein@Home search for continuous, nearly monochromatic, high-frequency gravitational waves in data from LIGO's fifth science run (S5). A number of searches have been carried out on LIGO data [1-6] targeting lower frequency ranges. The only other search covering frequencies up to $1500 \mathrm{~Hz}$ was conducted on S6 data [7] taken at least three years apart from the data used here. Our search results are only $33 \%$ less sensitive than those of Abbot et al. [7], even though the S5 data are less sensitive than the S6 data by more than a factor of 2. The search method presented here anticipates the procedure that will be used on the advanced detector (aLIGO) data.

This search can be considered an extension of the S5 Einstein@ Home search [1] although it employs a different

\footnotetext{
*avneet.singh@aei.mpg.de

†maria.alessandra.papa@aei.mpg.de
}

Published by the American Physical Society under the terms of the Creative Commons Attribution 3.0 License. Further distribution of this work must maintain attribution to the author(s) and the published article's title, journal citation, and DOI. search technique: this search uses the global correlation transform (GCT) method to combine results from coherent $\mathcal{F}$-statistic searches [8,9], as opposed to the previous Einstein@Home search [1] that employed the Houghtransform method to perform this combination. In the end, at fixed computing resources, these two search methods are comparable in sensitivity. However, a semicoherent $\mathcal{F}$-statistic search is more efficient when considering a broad spin-down range, and for the Einstein@ Home searches we have decided to adopt it as our "work horse."

We do not find any significant signal(s) among the set of searched waveforms. Thus, we set $90 \%$ confidence upper limits on continuous gravitational wave strain amplitudes; near the lower end of the search frequency range between 1253.217-1255.217 Hz, the most constraining upper limit is $5.0 \times 10^{-24}$, while toward the higher end of the search frequency range nearing $1500 \mathrm{~Hz}$, the upper-limit value is roughly $6.2 \times 10^{-24}$. Based on these upper limits, we can exclude certain combinations of signal frequency, star deformation (ellipticity) and distance values. We show with this search that even with S5 data from the first generation of $\mathrm{GW}$ detectors, such constraints do probe interesting regions of source parameter space.

\section{THE DATA}

The LIGO gravitational wave network consists of two detectors, $\mathrm{H} 1$ in Hanford (Washington) and L1 in Livingston (Louisiana), separated by a $3000-\mathrm{km}$ baseline. The S5 run lasted roughly two years between GPS time $815155213 \mathrm{sec}$ (Fri, Nov 04, 16:00:00 UTC 2005) and 875145614 sec (Sun, Sep 30, 00:00:00 UTC 2007). This 
search uses data spanning this observation period, and during this time, $\mathrm{H} 1$ and $\mathrm{L} 1$ had duty factors of $78 \%$ and $66 \%$, respectively $[10,11]$. The gaps in this data set are due to environmental or instrumental disturbances, or scheduled maintenance periods.

We follow [1,2], where the calibrated and high-pass filtered data from each detector are partitioned in 30-minute chunks and each chunk is Fourier-transformed after the application of a steep Tukey window. The set of short (time-baseline) Fourier transforms (SFT) that ensues is the input data for our search.

We further follow [1], where frequency bands known to contain spectral disturbances have been removed from the analysis. In fact, such data have been substituted with fake Gaussian noise at the same level as the neighboring undisturbed data; in Table III, we list these bands.

\section{THE SEARCH}

The search presented here is similar to the search on S6 data, reported in [6]. Our reference target signal is given by (1)-(4) in [4]; at emission, the signal is nearly monochromatic, typically with a small spin-down. The signal waveform in the detector data is modulated in frequency because of the relative motion between the compact object and the detector; a modulation in amplitude also occurs because of the variation of the sensitivity of the detector with time across the sky.

The most sensitive search technique that one could use is a fully coherent combination of the detectors' data, matched to the waveform that one is looking for. The (amplitude) sensitivity of such a method increases with the square root of the time span of the data used. However, the computational cost to resolve different waveforms increases very rapidly with increasing time span of the data, and this makes a fully coherent search over a large frequency range computationally unfeasible when using months of data. This is the main reason why semicoherent search methods have been developed. These methods perform coherent searches over shorter stretches of data, called segments, and then combine the results with incoherent techniques.

This search covers waveforms from the entire sky, with frequencies in a $250 \mathrm{~Hz}$ range from $1249.717 \mathrm{~Hz}$ to $1499.717 \mathrm{~Hz}$, and with a first-order spin-down between $-2.93 \times 10^{-9} \mathrm{~Hz} / \mathrm{s}$ and $5.53 \times 10^{-10} \mathrm{~Hz} / \mathrm{s}$, similar to previous Einstein@Home searches. We use a stack-slide semicoherent search procedure implemented with the GCT method [8,9]. The data is divided into $N_{\text {seg }}$ segments, each spanning $\mathrm{T}_{\text {coh }}$ in time. The coherent multi-detector $\mathcal{F}$-statistic [12] is computed on each segment for all the points on a coarse $\lambda_{\mathrm{c}} \equiv\left(f_{\mathrm{c}}, \dot{f}_{\mathrm{c}}, \alpha_{\mathrm{c}}, \delta_{\mathrm{c}}\right)$ signal waveform parameter grid, and then results from the individual segments are summed, one per segment, to yield the final core detection statistic $\overline{\mathcal{F}}$, as shown in (1); $\alpha, \delta$ are the equatorial sky coordinates of the source position, while $f$ and $\dot{f}$ are the frequency and first-order spin-down of the signal, respectively. Depending on which $\lambda_{\mathrm{c}}$ parameter points are taken on the coarse grid for each segment in this sum, the result will approximate the detection statistic computed on a $\lambda_{f}$ parameter point on a finer grid:

$$
\overline{\mathcal{F}}\left(\lambda_{f}\right):=\frac{1}{N_{\text {seg }}} \sum_{i=1}^{N_{\text {seg }}} \mathcal{F}\left(\lambda_{\mathrm{c}}^{i}\right) .
$$

In a stack-slide search in Gaussian noise, $N_{\text {seg }} \times 2 \overline{\mathcal{F}}$ follows a $\chi_{4 N_{\text {seg }}}^{2}$ chi-squared distribution with $4 N_{\text {seg }}$ degrees of freedom.

The most important search parameters are then $N_{\text {seg }}$, $\mathrm{T}_{\text {coh }}$, the signal parameter search grids $\lambda_{\mathrm{c}}, \lambda_{f}$, the total spanned observation time $\mathrm{T}_{\mathrm{obs}}$, and finally the ranking statistic used to rank parameter space cells i.e. $2 \overline{\mathcal{F}}$.

The grid spacing in frequency $\delta f$ and spin-down $\delta \dot{f}$ are constant over the search range. The same frequency spacing and sky grid are used for the coherent analysis and in the incoherent summing. The spin-down spacing of the incoherent analysis is finer by a factor of $\gamma$ with respect to that of the coherent analysis. In Table I, we summarize the search parameters.

The sky grid for the search is constructed by tiling the projected equatorial plane uniformly with squares of edge length $d_{\text {sky }}$. The length of the edge of the squares is a function of the frequency $f$ of the signal, and parametrized in terms of a so-called sky-mismatch parameter $\left(\mathrm{m}_{\text {sky }}\right)$ as

$$
d_{\mathrm{sky}}=\frac{1}{f} \frac{\sqrt{\mathrm{m}_{\mathrm{sky}}}}{\pi \tau_{\mathrm{E}}},
$$

where $\tau_{\mathrm{E}}=0.021$ seconds and $\mathrm{m}_{\text {sky }}=0.3$, also given in Table I. The sky grids are constant over $10 \mathrm{~Hz}$-wide frequency bands, and are calculated for the highest frequency in the band. In Fig. 1, we illustrate an example of the sky grid. The total number of templates in $50 \mathrm{mHz}$ bands as a function of frequency is shown in Fig. 2. This

TABLE I. Search parameters for the search. $t_{\text {ref }}$ is the reference time that defines the frequency and spin-down values.

\begin{tabular}{lc}
\hline \hline Quantity & Value \\
\hline $\mathrm{T}_{\text {coh }}$ (hours) & 30.0 \\
$\mathrm{~T}_{\text {obs }}$ (days) & 653.18 \\
$t_{\text {ref }}(\mathrm{GPS}$ seconds) & 847063082.5 \\
$N_{\text {seg }}$ & 205 \\
$\delta f_{\mathrm{c}}(\mathrm{Hz})$ & $6.71 \times 10^{-6}$ \\
$\delta \dot{f}_{\mathrm{c}}(\mathrm{Hz} / \mathrm{s})$ & $5.78 \times 10^{-10}$ \\
$\gamma$ & 1399 \\
$\mathrm{~m}_{\text {sky }}$ & 0.30 \\
\hline \hline
\end{tabular}



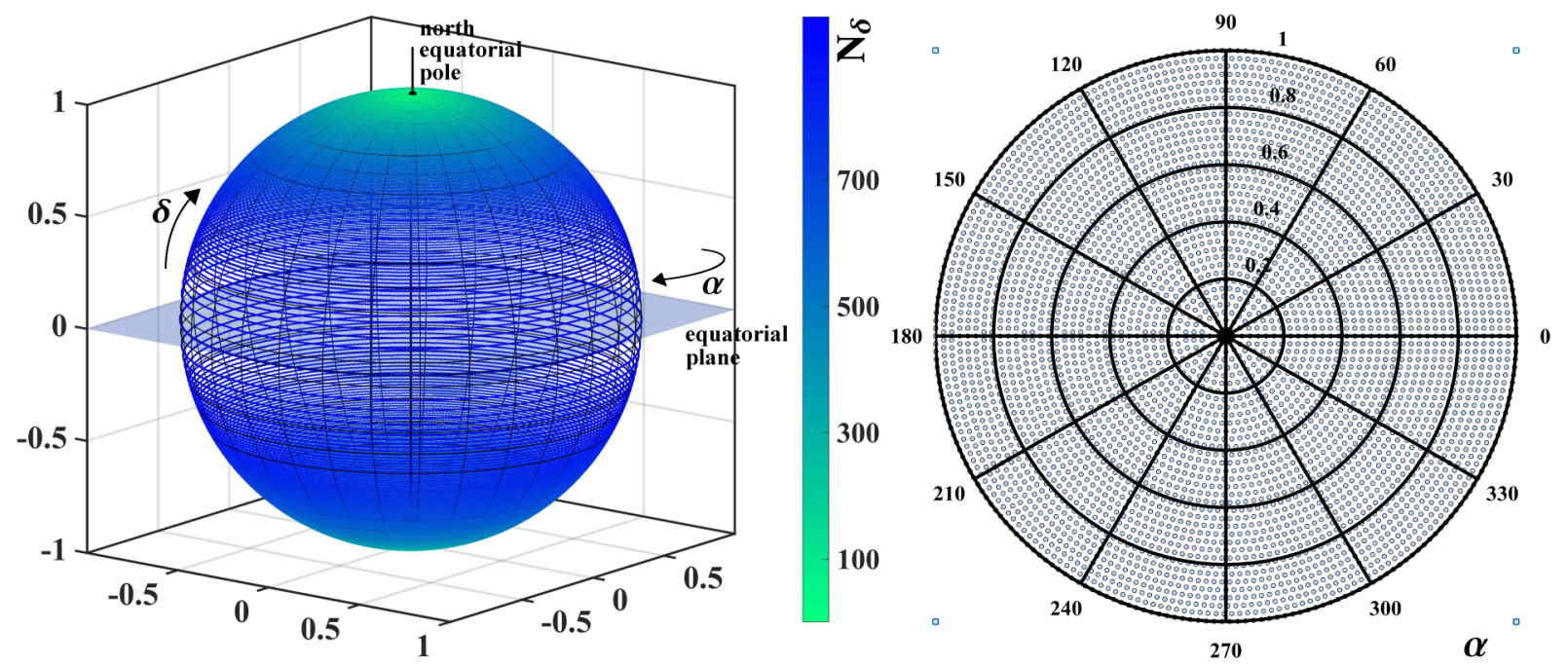

FIG. 1. Tilling of sky grid for the frequency band $1240-1250 \mathrm{~Hz} ; d_{\text {sky }}=6.6 \times 10^{-4}$ for this band. In the left panel, we show the skygrid points on the celestial sphere; the color-code traces the number of sky-grid points, $\mathrm{N}_{\delta}$, as a function of equatorial latitude $\delta$. The right panel is a polar plot of the northern equatorial hemisphere of the same sky grid but with density scaled down by a factor of 4 to allow for better viewing. In the polar plot, $\theta=\alpha$ and $r=\cos (\delta)$.

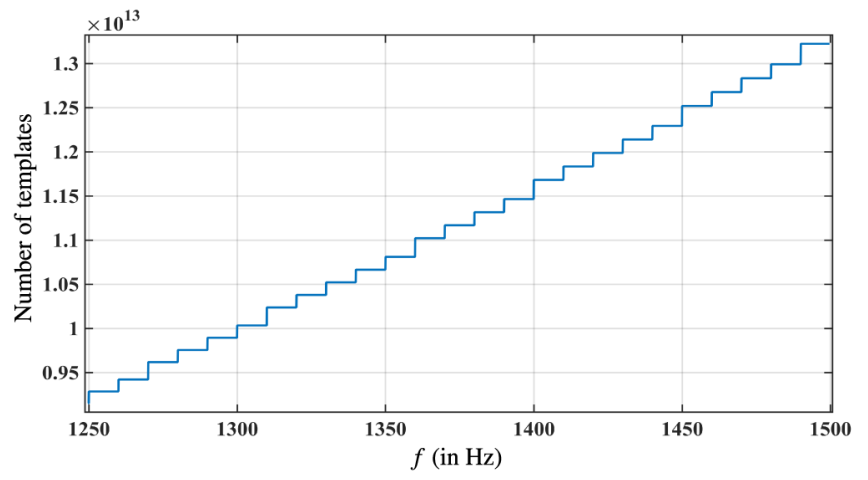

FIG. 2. Number of templates searched in $50 \mathrm{mHz}$ bands. The variation in template count arises from the variation is number of sky-grid points every $10 \mathrm{~Hz}$ in frequency. Each $50 \mathrm{mHz}$ band contributes roughly $6.3 \times 10^{7}$ templates in frequency and spindown (on the finer grid refined by refinement factor $\gamma$.)

search explores a total of $5.6 \times 10^{16}$ waveform templates across the $\lambda_{f} \equiv\left(f_{f}, \dot{f}_{f}, \alpha_{f}, \delta_{f}\right)$ parameter space.

The search is divided into work-units (WU), each searching a very small subset of template waveforms. The WU are sent to Einstein@Home volunteers and each WU occupies the volunteer/host computer for roughly 6 hours. One such WU covers a $50 \mathrm{mHz}$ band, the entire spin-down range, and 139-140 points in the sky. It is necessary to have 6.4 million different WU to cover the whole parameter space. Each WU returns a ranked list of the most significant $10^{4}$ candidates found in the parameter space that is searched.

\section{IDENTIFICATION OF UNDISTURBED BANDS}

In Table III, we list the central frequencies and bandwidths of SFT data known to contain spectral lines from instrumental artifacts. These frequency regions were identified before the Einstein@Home run, and we were able to replace the corresponding data with Gaussian noise matching the noise level of neighboring quiet bands.

Consequently, some search results have contributions from this "fake data." The intervals in signal-frequency where the search results come entirely from fake data are indicated as "All fake data" in Table IV. In these intervals of signal-frequency, we effectively do not have search results. The other three columns in Table IV provide signal-frequency intervals where results might have contributions from fake data. In these regions, depending on the signal parameters, the detection efficiency might be affected.

Despite the removal of known disturbances from the data, it still contains unknown noise artifacts producing $2 \overline{\mathcal{F}}$ values that do not follow the expected distribution for Gaussian noise. These artifacts usually have narrowband characteristics; we identify such "disturbed" signalfrequency intervals in the search results and exclude them from further consideration. The benefit of such exclusions is that, in the remaining "undisturbed" bands, we can rely on semianalytic predictions for the significance of the observed $2 \overline{\mathcal{F}}$ values, and we can set a uniform detection criterion across the entire parameter space. It is true that we forego the possibility of detecting a signal in the disturbed frequency intervals. However, in order to perform reliable analyses in these intervals, ad-hoc studies and tuning of the procedures would need to be performed on each disturbed band separately and these would be very time consuming. Since the undisturbed intervals in data comprise over $95 \%$ of the total data, we believe that ignoring the disturbed bands for this search is a reasonable choice. In the future, a 

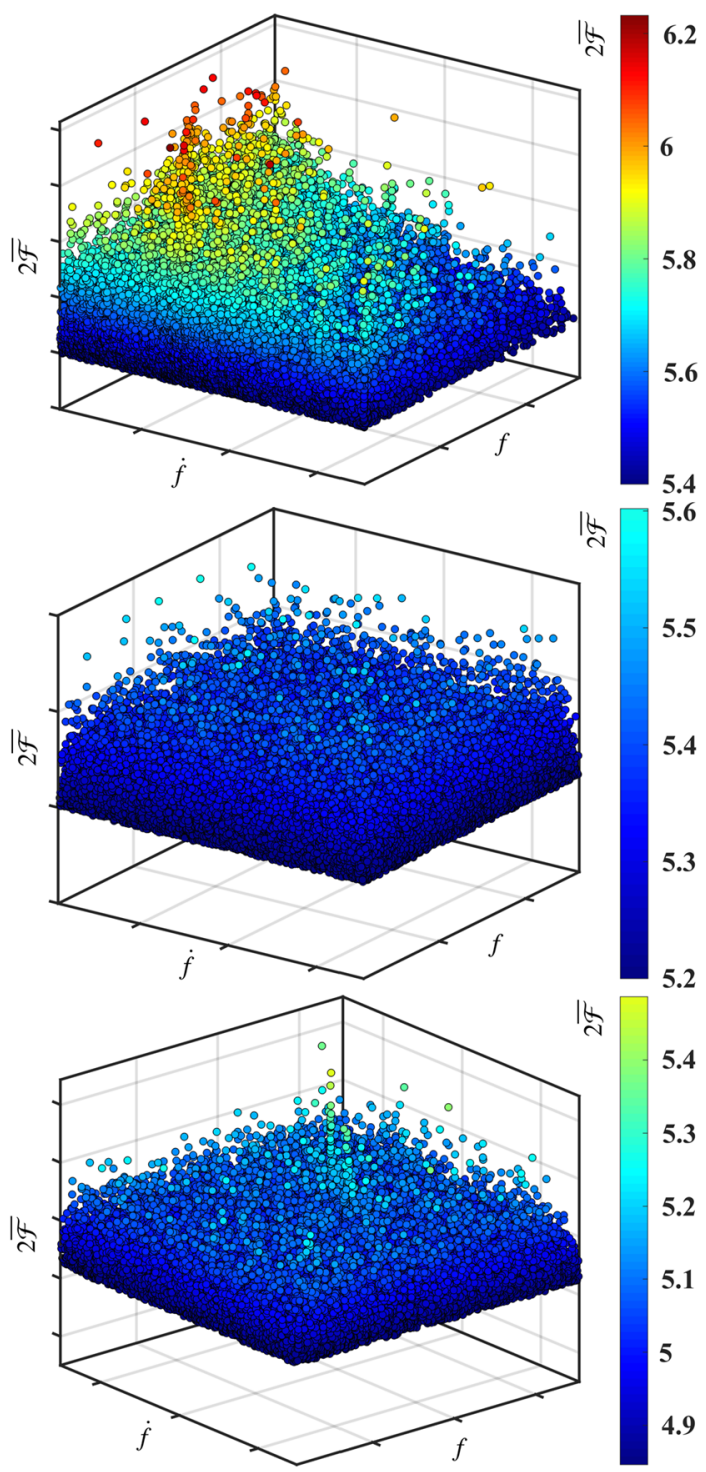

FIG. 3. We plot the color-coded $2 \overline{\mathcal{F}}$ values on the $z$ axis in three $50 \mathrm{mHz}$ bands. The top-most band is marked as "disturbed"; the middle band is an example of an "undisturbed" band; the bottommost band is an example of an undisturbed band but containing a simulated continuous gravitational wave signal.

focused effort on the analysis of the disturbed bands could attempt to recover some sensitivity in those regions.

The identification of undisturbed bands is carried out via a visual inspection method. This visual inspection of the data is performed by two scientists who look at various distributions of the $2 \overline{\mathcal{F}}$ values in the $(f, \dot{f})$ parameter space in $50 \mathrm{mHz}$ bands. They rank these $50 \mathrm{mHz}$ bands with 4 numbers: $0,1,2,3$; a 0 ranking marks the band as "undisturbed," a 3 ranks the band as "disturbed," and rankings of 1 or 2 mark the band as "marginally disturbed." A $50 \mathrm{mHz}$ band is eventually considered to be undisturbed if it is marked as 0 by both scientists. The criteria used for this inspection are based on training sets of real data containing simulated signals. These criteria are designed to

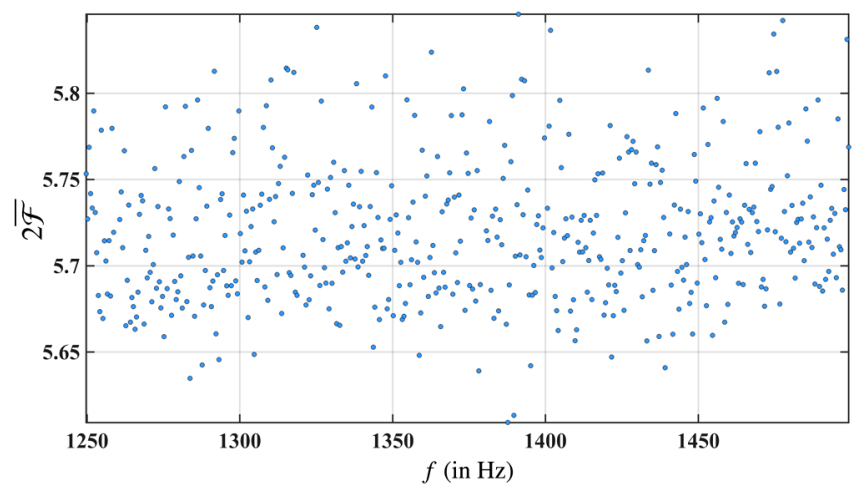

FIG. 4. Highest values of $2 \overline{\mathcal{F}}$ in every $0.5 \mathrm{~Hz}$ band as a function of starting frequency of the band.

exclude disturbed set of results while retaining data sets with signal-like properties, and to err on the conservative side in terms of not falsely dismissing signals. A significant part of this visual inspection work can be automated [13], but at the time of this search, the procedure had not been fully tested and tuned. In Fig. 3, we empirically illustrate these criteria using three examples. Following this procedure, $3 \%$ of the total $500050 \mathrm{mHz}$ bands are marked as "disturbed" by visual inspection. These excluded bands are listed in Table V (type D), together with the $50 \mathrm{mHz}$ bands excluded as a result of the cleaning of known disturbances above (type C), i.e. marked as "All fake data" in Table IV. In consequence to these exclusions, there exist $0.5 \mathrm{~Hz}$ bands comprising results from less than ten $50 \mathrm{mHz}$ bands. We define "fill level" as the percentage of $50 \mathrm{mHz}$ bands that contribute to the results in $0.5 \mathrm{~Hz}$ intervals, where $100 \%$ fill level signifies contribution by all ten $50 \mathrm{mHz}$ bands. In Fig. 7, we show the distribution of fill levels for the $0.5 \mathrm{~Hz}$ bands searched.

In Fig. 4, we plot the loudest observed candidate i.e. the candidate with the highest $2 \overline{\mathcal{F}}$ value in each $0.5 \mathrm{~Hz}$ band of the search frequency range. The loudest candidate in our search has a detection-statistic value of $2 \overline{\mathcal{F}}=5.846$ at a frequency of roughly $1391.667 \mathrm{~Hz}$. In order to determine the significance of this loudest candidate, we compare it to the expected value for the highest detection statistic in our search. In order to determine this expected value, we have to estimate the number of independent trials performed in the search i.e. total number of independent realizations of our detection statistic $2 \overline{\mathcal{F}}$.

The number of independent realizations of the detection statistic, $N_{\text {trials }}$, in a search through a bank of signal templates is smaller than the total number of searched templates, $N_{\text {templates }}$. We estimate $N_{\text {trials }}$ as a function of frequency in $10 \mathrm{~Hz}$ frequency intervals. In each of these $10 \mathrm{~Hz}$ intervals, we fit the distribution of loudest candidates from $50 \mathrm{mHz}$ bands to the expected distribution [14], and obtain the best-fit value of $N_{\text {trials }}$. We perform this calculation in $10 \mathrm{~Hz}$ intervals since the sky grids, along with $N_{\text {templates, }}$, are constant over $10 \mathrm{~Hz}$ frequency intervals. In 
RESULTS OF AN ALL-SKY HIGH-FREQUENCY ...

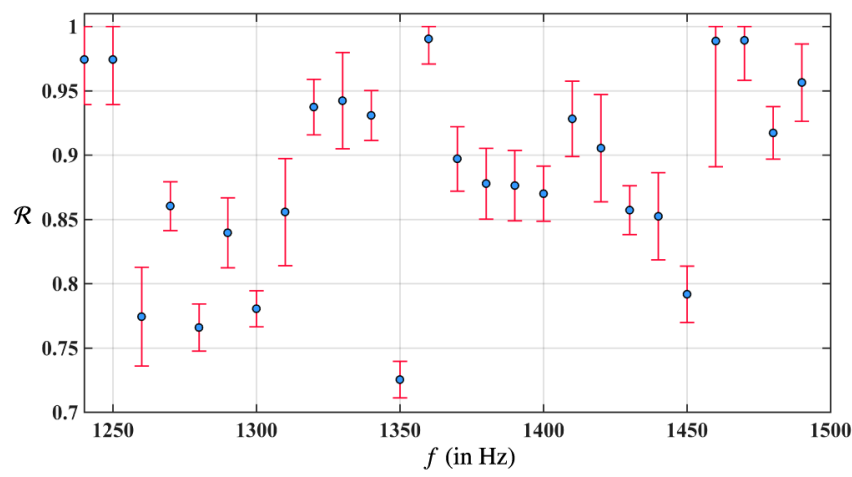

FIG. 5. Ratio $\mathcal{R}=N_{\text {trials }} / N_{\text {templates }}$ as a function of frequency in $10 \mathrm{~Hz}$ intervals. The error bars represent the 1- $\sigma$ statistical errors from the fitting procedure described in the text.

Fig. 5, we plot the ratio $\mathcal{R}=N_{\text {trials }} / N_{\text {templates }}$ as a function of frequency.

With $\mathcal{R}(f)$ in hand, we evaluate the expected value for the loudest detection statistic ( $\left.2 \overline{\mathcal{F}}_{\text {exp }}\right)$ in $0.5 \mathrm{~Hz}$ bands, and the standard deviation $\left(\sigma_{\text {exp }}\right)$ of the associated distribution using (5)-(6) of [14], with $N_{\text {seg }}=205$ and $N_{\text {trials }}=$ $\mathcal{R} N_{\text {templates. }}$. Based on these values, we can estimate the
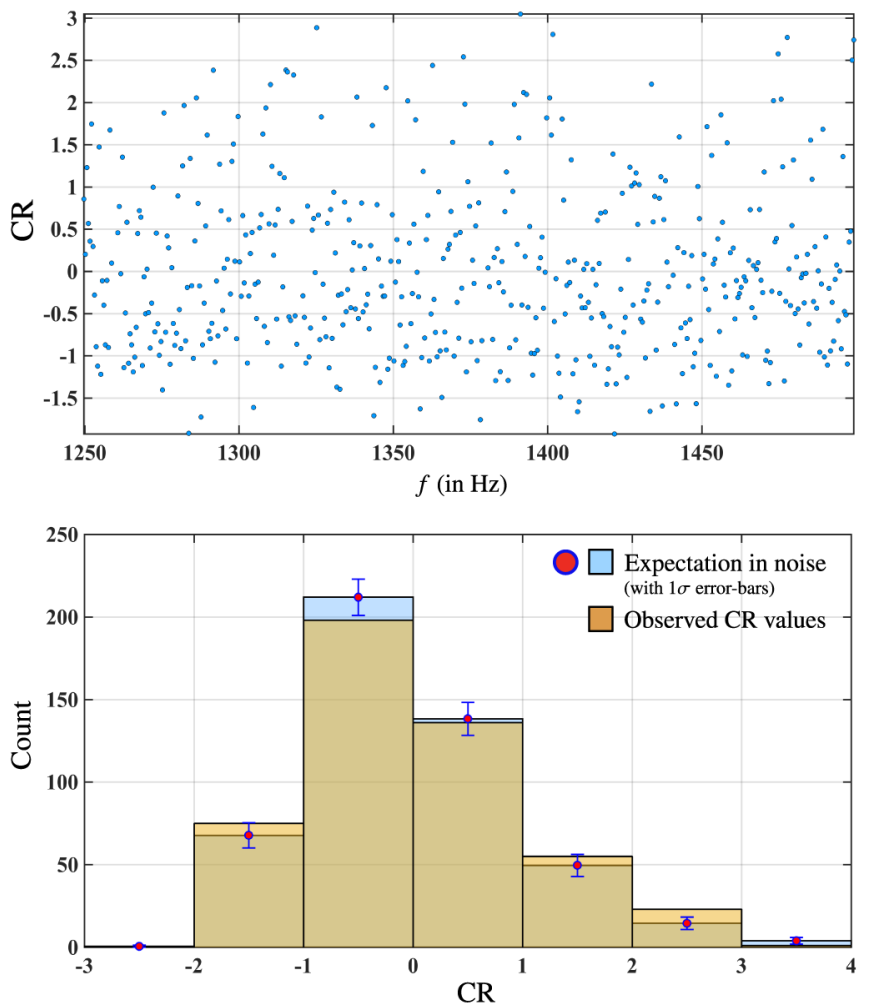

FIG. 6. In the top panel, we plot the significance of the loudest observed candidate in every $0.5 \mathrm{~Hz}$ band as a function of starting frequency of the band. In the bottom panel, we show the observed distribution of CR values (top brown histogram bars), and the expected distribution of $\mathrm{CR}$ values for pure noise for reference (bottom blue histogram bars with markers). The significance folds in the expected value for the loudest $2 \overline{\mathcal{F}}$ and its standard deviation.
PHYSICAL REVIEW D 94, 064061 (2016)

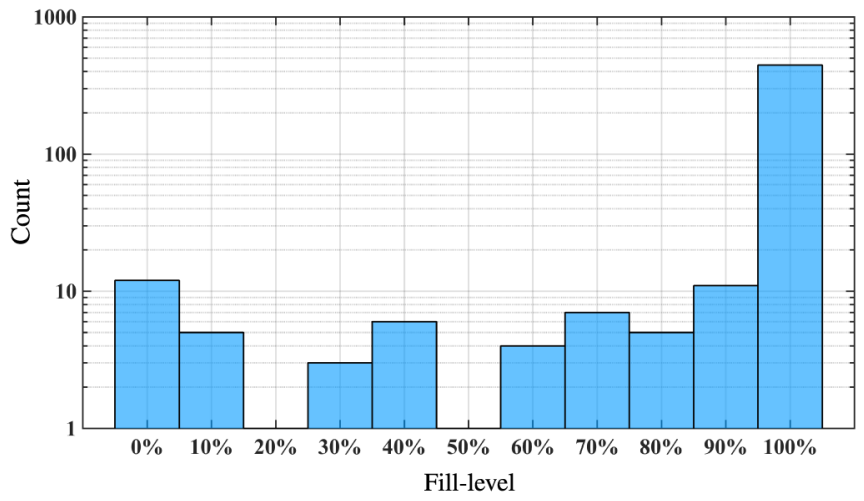

FIG. 7. Distribution of fill levels of $0.5 \mathrm{~Hz}$ bands.

significance of the observed loudest candidates (denoted by $\left.2 \overline{\mathcal{F}}_{\text {Loud }}\right)$ as the critical ratio (CR),

$$
\mathrm{CR}:=\frac{2 \overline{\mathcal{F}}_{\text {Loud }}-2 \overline{\mathcal{F}}_{\text {exp }}}{\sigma_{\text {exp }}}
$$

In Fig. 6, we plot the $\mathrm{CR}$ values of the observed loudest candidates in $0.5 \mathrm{~Hz}$ bands as a function of frequency (top panel) and their distribution (bottom panel).

In this search, the overall loudest candidate with $2 \overline{\mathcal{F}}=5.846$ is also the most significant candidate, with $\mathrm{CR}=3.05$. A deviation of $3.05 \sigma$ from the expected $2 \overline{\mathcal{F}}$ value would not be significant enough to claim a detection if we had only searched a single $0.5 \mathrm{~Hz}$ band. It is even less significant considering the fact that a total of $4850.5 \mathrm{~Hz}$ bands were searched.

We define the $p$-value associated with a $\mathrm{CR}$ as the probability of observing that particular value of CR or higher by random chance in a search over one $0.5 \mathrm{~Hz}$ band, performed over $N_{\text {trials }}$ independent trials using $N_{\text {seg }}$ segments. In Fig. 8, we see that the distribution of $p$-values associated with the loudest observed candidates in $0.5 \mathrm{~Hz}$ bands is consistent with what we expect from the

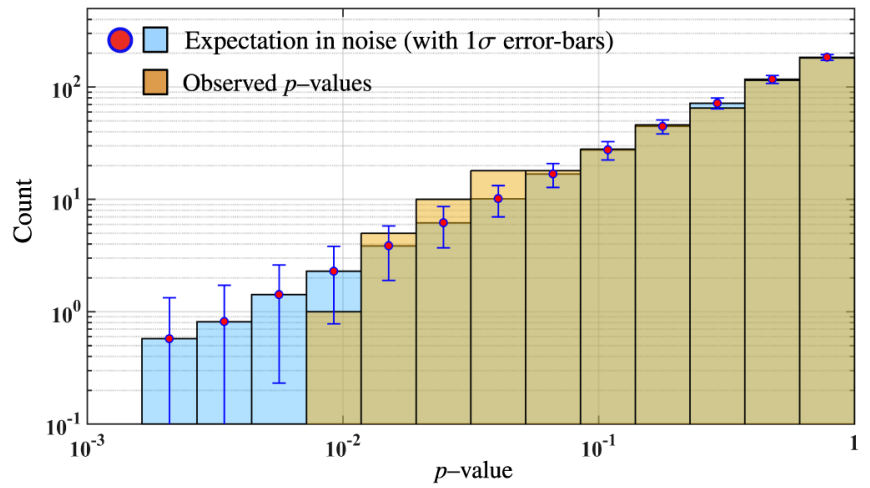

FIG. 8. $p$-values for the loudest observed candidates in $0.5 \mathrm{~Hz}$ bands in the data (top brown histogram bars), and the expected distribution of $p$-values for pure noise for reference (bottom blue histogram bars with markers). 


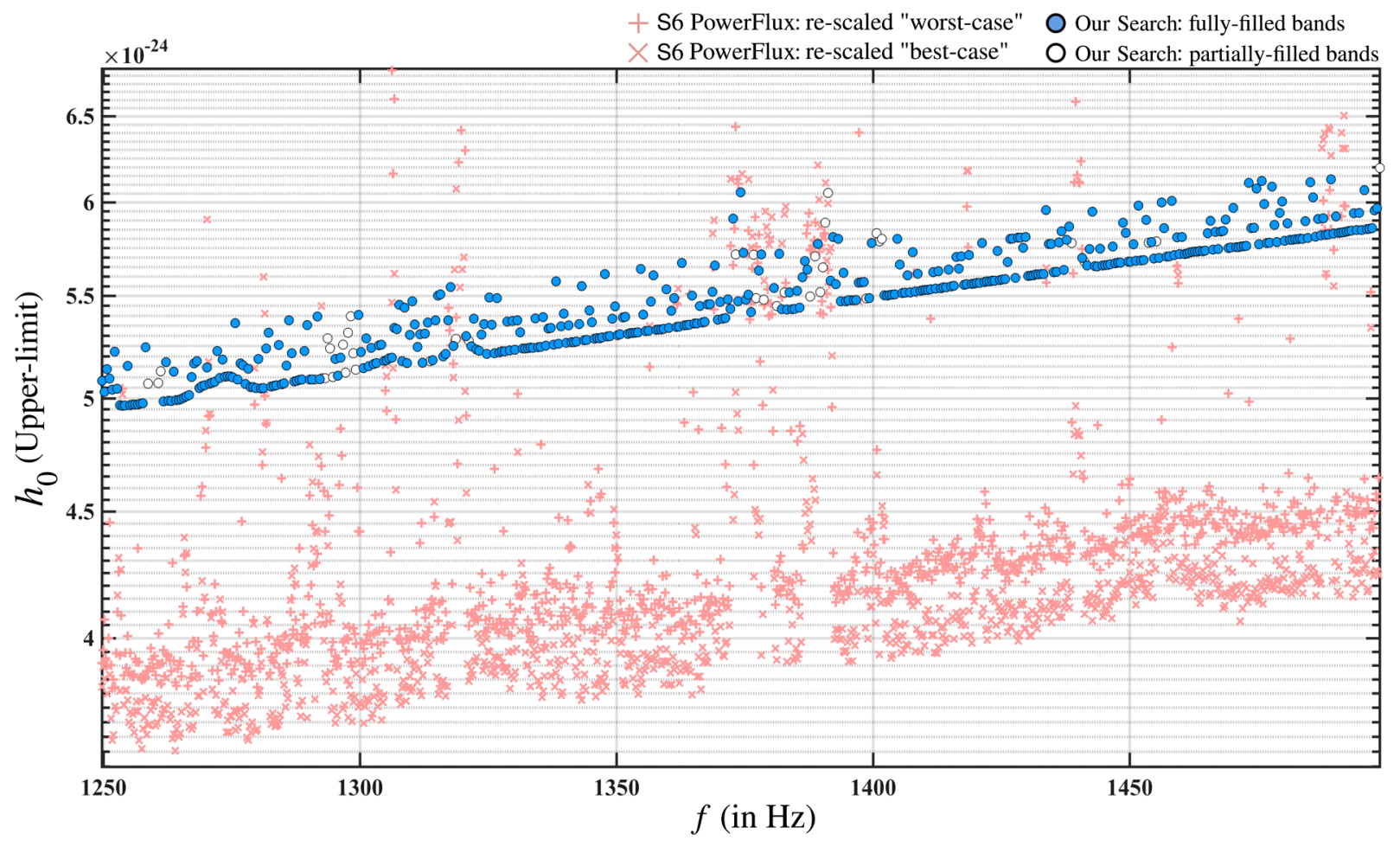

FIG. 9. The $90 \%$ confidence upper limits on the gravitational wave amplitude for signals with frequency within $0.5 \mathrm{~Hz}$ bands, over the entire sky, and within the spin-down range of the search described in Sec. III. The empty circular markers denote $0.5 \mathrm{~Hz}$ bands where the upper-limit value does not hold for all frequencies in that interval; the list of corresponding excluded frequencies is given in Table IV. For reference, we also plot the upper-limit results (with noncircular markers) from the only other high-frequency search [7], on significantly more sensitive S6 data. It should be noted that the upper limits from the PowerFlux search [7] are set at 95\% confidence rather than $90 \%$ confidence level as in this search, but refer to $0.25 \mathrm{~Hz}$ bands rather than $0.5 \mathrm{~Hz}$ bands.

noise-only scenario across the explored parameter space. In particular, we see in Fig. 8 that across $4850.5 \mathrm{~Hz}$ bands searched by our setup, we expect $2.3 \pm 1.5$ candidates at least as significant as $\mathrm{CR}=3.05$ ( $p$-value bin $10^{-2}$ for that band) by random chance, which makes our observed loudest candidate completely consistent with expectations from the noise-only case.

\section{UPPER LIMITS}

Our search results do not deviate from the expectations from noise-only data. Hence, we set frequentist upper limits on the maximum gravitational wave amplitude, $h_{0}^{90 \%}$, from the target source population consistent with this null result at $90 \%$ confidence in $0.5 \mathrm{~Hz}$ bands. Here, $h_{0}^{90 \%}$ is the gravitational wave amplitude for which $90 \%$ of the target population of signals would have produced a value of the detection statistic higher than the observed value.

Ideally, in order to estimate the $h_{0}^{90 \%}$ values in each $0.5 \mathrm{~Hz}$ band across the $250 \mathrm{~Hz}$ signal-frequency search range, we would perform Monte Carlo injection-andrecovery simulations in each of those bands. However, this is computationally very intensive. Therefore, we perform Monte Carlo simulations in six $0.5 \mathrm{~Hz}$ bands spread evenly across the $250 \mathrm{~Hz}$-wide frequency range, and in each of these six bands labeled by the index $k$, we estimate the $h_{0, \mathrm{CR}}^{90 \%}$ upper-limit value corresponding to eight different $\mathrm{CR}_{i}$ significance bins for the putative observed loudest candidate: $(0.0,0.5,1.0,1.5,2.0,2.5,3.0,3.5)$. In each of these six bands and for each of the eight detection criteria, we calculate the so-called "sensitivity depth," defined in [14]: $\mathcal{D}_{\mathrm{CR}_{i}}^{90 \%, k}$. Lastly, we average these sensitivity depths over the six bands and derive the average sensitivity depth $\mathcal{D}_{\mathrm{CR}_{i}}^{90 \%}$ for each detection criterion. The values of the sensitivity depths range between $\mathcal{D}_{\mathrm{CR}_{0.0}}^{90 \%}=30.6 \mathrm{~Hz}^{-1 / 2}$ and $\mathcal{D}_{\mathrm{CR}_{3.5}}^{90 \%}=28.8 \mathrm{~Hz}^{-1 / 2}$. We use these $\mathcal{D}_{\mathrm{CR}_{i}}^{90 \%}$ values to set upper limits in the bands (labeled by $j$ ) where we have not performed any Monte Carlo simulations as follows,

$$
h_{0}^{90 \%}\left(f_{j}\right)=\frac{\sqrt{S_{h}\left(f_{j}\right)}}{\mathcal{D}_{\mathrm{CR}_{i(j)}}^{90 \%}},
$$

where $\mathrm{CR}_{i}(j)$ is the significance bin $i$ corresponding to the loudest observed candidate in the $j$ th frequency band, and $S_{h}\left(f_{j}\right)$ is the average amplitude spectral density of the data in that band, measured in $\mathrm{Hz}^{-1 / 2}$. The uncertainties on the $h_{0}^{90 \%}$ upper-limit values introduced by this procedure amount to roughly $10 \%$ of the nominal $h_{0}^{90 \%}$ upper-limit value. The final $h_{0}^{90 \%}$ upper-limit values for this search, 

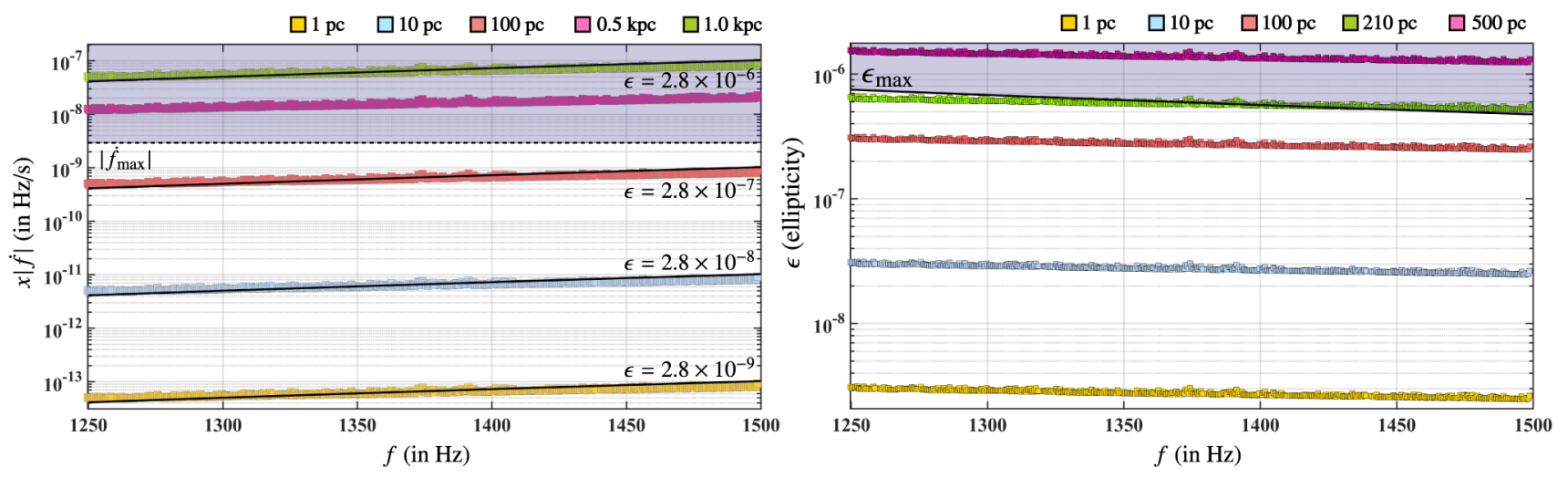

FIG. 10. Gravitational wave amplitude upper limits recast as curves in the $(f, x|\dot{f}|)$ plane (left panel) for sources at given distances, where $f$ is the signal frequency and $x|\dot{f}|$ is the gravitational wave spin-down i.e. the fraction of the actual spin-down $|\dot{f}|$ that accounts for the rotational energy loss due to gravitational wave emission. We have superimposed the curves of constant ellipticity $\epsilon$. The dotted line at $\left|\dot{f}_{\max }\right|$ indicates the maximum magnitude of searched spin-down, namely $2.93 \times 10^{-9} \mathrm{~Hz} / \mathrm{s}$. The right panel shows the corresponding $(f, \epsilon)$ upper-limit curves for sources at various distances. The $\epsilon_{\max }=41.3 \times f^{-5 / 2}$ curve is the ellipticity corresponding to the highest $|\dot{f}|$ searched.

including an additional 10\% calibration uncertainty, are given in Table II and shown in Fig. 9.

Note that we do not set upper limits in $0.5 \mathrm{~Hz}$ bands where the results are entirely produced with fake Gaussian data inserted by the cleaning procedure described in Sec. IV; $h_{0}^{90 \%}$ upper-limit values for such bands do not appear either in Table II, or in Fig. 9. Moreover, there also exist $50 \mathrm{mHz}$ bands that contain results contributed by entirely fake data as a result of the cleaning procedure, or that have been excluded from the analysis because they are marked as disturbed by the visual inspection method described in Sec. IV. We mark the $0.5 \mathrm{~Hz}$ bands which host these particular $50 \mathrm{mHz}$ bands with empty circles in Fig. 9. In Table V, we provide a complete list of such $50 \mathrm{mHz}$ bands, highlighting that the upper-limit values do not apply to these bands. Finally, we note that, because of the cleaning procedure, there exist signal-frequency bands where the search results may have contributions from some fake data. We list these signalfrequency ranges in Table IV. In line with the remarks in Sec. IV, and for the sake of completeness, Table IV also contains the cleaned bands featured under type $\mathrm{C}$ in Table V, under the column header "All fake data."

\section{CONCLUSIONS}

This search did not yield any evidence of continuous gravitational waves in the LIGO fifth science run data in the high-frequency range of $1250-1500 \mathrm{~Hz}$. The lowest value for the upper limit is $5.0 \times 10^{-24}$ for signal frequencies between 1253.217-1255.217 Hz. We show in Fig. 9 that these $h_{0}^{90 \%}$ upper limits are about $33 \%$ higher than the upper limits ${ }^{1}$ set [7] in the same frequency range but using S6

\footnotetext{
${ }^{1}$ The upper-limit values of [7] have been rescaled according to [15] in order to allow a direct comparison with our $h_{0}^{90 \%}$ upperlimit results.
}

data. In this frequency range, the $\mathrm{S} 6$ run data are about a factor 2.4 more sensitive compared to the S5 data used in this search. We can express the $h_{0}^{90 \%}$ upper limits as bounds on the maximum distance from Earth within which we can exclude a rotating compact object emitting continuous gravitational waves at a given frequency $f$ due to a fixed and nonaxisymmetric mass quadrupole moment, characterized by $\epsilon I$, with $I$ being the principal moment of inertia, and $\epsilon$ the ellipticity of the object. The GW spindown is the fraction of spin-down, $x|\dot{f}|$, responsible for continuous gravitational wave emission [16]. The ellipticity $\epsilon$ of the compact object necessary to sustain such emission is given by

$$
\epsilon(f, x|\dot{f}|)=\sqrt{\frac{5 c^{5}}{32 \pi^{4} G} \frac{x|\dot{f}|}{I f^{5}}},
$$

where $c$ is the speed of light, $G$ is the gravitational constant. Moreover, since the gravitational wave amplitude for an object at a distance $d$, with an ellipticity $\epsilon$ given by (5), is expressed as

$$
h_{0}(f, x|\dot{f}|, d)=\frac{1}{d} \sqrt{\frac{5 I G}{2 c^{3}} \frac{x \mid \dot{f}}{f}},
$$

we can recast the $h_{0}^{90 \%}$ upper-limit curves as $(f, x|\dot{f}|)$ curves, or as $(f, \epsilon)$ curves, both parametrized by different values of the distance $d$, as shown in Fig. 10. We find that within 100 pc of Earth, our upper limits exclude objects with ellipticities higher than roughly $2.8 \times 10^{-7}\left[\frac{10^{38} \mathrm{~kg} \mathrm{~m}^{2}}{I}\right]$, corresponding to $\mathrm{GW}$-spindown values between roughly $4.0 \times 10^{-10}$ and $1.0 \times 10^{-9} \mathrm{~Hz} / \mathrm{s}$. This value is well below the maximum elastic deformation that a relativistic star could sustain; see [17] and references therein. 
The search presented here is probably the last all-sky search on S5 data, and by inspecting the higher frequency range for continuous gravitational wave emission, it concludes the Einstein @ Home observing campaign on this data. Consistent with the recent results on S6 data [7], we also find no continuous GW signal in the S5 data. However, mechanisms for transient or intermittent GW emission have been proposed [18-20] which would not a priori exclude a signal that is "ON" during the S5 run and "OFF" during the S6 run. The estimates for the time scales, frequencies and spindowns of continuous gravitational wave signals from isolated neutron stars lasting weeks to months span a very broad range of values - orders of magnitude. There are several different mechanisms that could sustain such emission at a level that this search could have detected, and with spin-down values consistent with the total energy emitted in the process, and with the spin-down range spanned by this search.

\section{ACKNOWLEDGMENTS}

Maria Alessandra Papa, Bruce Allen, and Xavier Siemens gratefully acknowledge the support from NSF PHY Grant No. 1104902. All the postprocessing computational work for this search was carried out on the ATLAS supercomputing cluster at the Max Planck Institut für Gravitationsphysik/ Leibniz Universität Hannover. We also acknowledge the Continuous Wave Group of the LIGO Scientific Collaboration for useful discussions and, in particular, its chair, Keith Riles, for his careful reading of the manuscript. This paper has been assigned LIGO Document No. P1600196.

\section{APPENDIX: TABULAR DATA}

See Tables II-V.

\section{Upper-limit $\boldsymbol{h}_{0}^{90 \%}$ values}

See Table II.

TABLE II. Left column denotes the starting frequency of each $0.5 \mathrm{~Hz}$ signal-frequency band in which we set upper limits; right column states the upper-limit value i.e. $h_{0}^{90 \%}$, for that $0.5 \mathrm{~Hz}$ band. Note: the $h_{0}^{90 \%}$ values quoted here include additional $10 \%$ uncertainty introduced by data calibration procedure.

\begin{tabular}{|c|c|c|c|c|c|c|c|}
\hline$f($ in $\mathrm{Hz})$ & $h_{0}^{90 \%} \times 10^{24}$ & $f($ in $\mathrm{Hz})$ & $h_{0}^{90 \%} \times 10^{24}$ & $f($ in $\mathrm{Hz})$ & $h_{0}^{90 \%} \times 10^{24}$ & $f($ in $\mathrm{Hz})$ & $h_{0}^{90 \%} \times 10^{24}$ \\
\hline 1249.717 & $5.1 \pm 1.0$ & 1250.217 & $5.0 \pm 1.0$ & 1250.717 & $5.1 \pm 1.0$ & 1251.217 & $5.1 \pm 1.0$ \\
\hline 1251.717 & $5.0 \pm 1.0$ & 1252.217 & $5.2 \pm 1.1$ & 1252.717 & $5.0 \pm 1.0$ & 1253.217 & $5.0 \pm 0.9$ \\
\hline 1253.717 & $5.0 \pm 0.9$ & 1254.217 & $5.0 \pm 0.9$ & 1254.717 & $5.2 \pm 1.0$ & 1255.217 & $5.0 \pm 0.9$ \\
\hline 1255.717 & $5.0 \pm 0.9$ & 1256.217 & $5.0 \pm 0.9$ & 1256.717 & $5.0 \pm 0.9$ & 1257.217 & $5.0 \pm 0.9$ \\
\hline 1257.717 & $5.0 \pm 0.9$ & 1258.217 & $5.2 \pm 1.1$ & 1258.717 & $5.1 \pm 1.0$ & 1260.717 & $5.1 \pm 1.0$ \\
\hline 1261.217 & $5.1 \pm 1.0$ & 1261.717 & $5.0 \pm 0.9$ & 1262.217 & $5.2 \pm 1.0$ & 1262.717 & $5.0 \pm 0.9$ \\
\hline 1263.217 & $5.0 \pm 0.9$ & 1263.717 & $5.1 \pm 1.0$ & 1264.217 & $5.0 \pm 0.9$ & 1264.717 & $5.0 \pm 0.9$ \\
\hline 1265.217 & $5.0 \pm 0.9$ & 1265.717 & $5.0 \pm 0.9$ & 1266.217 & $5.0 \pm 0.9$ & 1266.717 & $5.0 \pm 0.9$ \\
\hline 1267.217 & $5.1 \pm 1.0$ & 1267.717 & $5.2 \pm 1.0$ & 1268.217 & $5.2 \pm 1.0$ & 1268.717 & $5.0 \pm 0.9$ \\
\hline 1269.217 & $5.1 \pm 0.9$ & 1269.717 & $5.1 \pm 0.9$ & 1270.217 & $5.1 \pm 1.0$ & 1270.717 & $5.1 \pm 0.9$ \\
\hline 1271.217 & $5.1 \pm 0.9$ & 1271.717 & $5.1 \pm 0.9$ & 1272.217 & $5.2 \pm 1.0$ & 1272.717 & $5.1 \pm 0.9$ \\
\hline 1273.217 & $5.2 \pm 1.0$ & 1273.717 & $5.1 \pm 0.9$ & 1274.217 & $5.1 \pm 0.9$ & 1274.717 & $5.1 \pm 0.9$ \\
\hline 1275.217 & $5.1 \pm 0.9$ & 1275.717 & $5.4 \pm 1.1$ & 1276.217 & $5.1 \pm 0.9$ & 1276.717 & $5.2 \pm 1.0$ \\
\hline 1277.217 & $5.2 \pm 1.0$ & 1277.717 & $5.1 \pm 0.9$ & 1278.217 & $5.1 \pm 1.0$ & 1278.717 & $5.1 \pm 0.9$ \\
\hline 1279.217 & $5.1 \pm 0.9$ & 1279.717 & $5.0 \pm 0.9$ & 1280.217 & $5.2 \pm 1.0$ & 1280.717 & $5.0 \pm 0.9$ \\
\hline 1281.217 & $5.0 \pm 0.9$ & 1281.717 & $5.2 \pm 1.0$ & 1282.217 & $5.3 \pm 1.1$ & 1282.717 & $5.1 \pm 0.9$ \\
\hline 1283.217 & $5.1 \pm 0.9$ & 1283.717 & $5.1 \pm 0.9$ & 1284.217 & $5.3 \pm 1.0$ & 1284.717 & $5.1 \pm 0.9$ \\
\hline 1285.217 & $5.1 \pm 0.9$ & 1285.717 & $5.2 \pm 1.0$ & 1286.217 & $5.4 \pm 1.1$ & 1286.717 & $5.2 \pm 1.0$ \\
\hline 1287.217 & $5.1 \pm 0.9$ & 1287.717 & $5.1 \pm 0.9$ & 1288.217 & $5.1 \pm 0.9$ & 1288.717 & $5.1 \pm 0.9$ \\
\hline 1289.217 & $5.2 \pm 1.0$ & 1289.717 & $5.4 \pm 1.1$ & 1290.217 & $5.1 \pm 0.9$ & 1290.717 & $5.1 \pm 0.9$ \\
\hline 1291.217 & $5.1 \pm 0.9$ & 1291.717 & $5.4 \pm 1.1$ & 1292.217 & $5.1 \pm 0.9$ & 1292.717 & $5.1 \pm 0.9$ \\
\hline 1293.217 & $5.1 \pm 0.9$ & 1293.717 & $5.3 \pm 1.0$ & 1294.217 & $5.2 \pm 1.0$ & 1294.717 & $5.1 \pm 0.9$ \\
\hline 1295.217 & $5.2 \pm 1.0$ & 1295.717 & $5.1 \pm 0.9$ & 1296.217 & $5.2 \pm 1.0$ & 1296.717 & $5.3 \pm 1.0$ \\
\hline 1297.217 & $5.1 \pm 0.9$ & 1297.717 & $5.3 \pm 1.0$ & 1298.217 & $5.4 \pm 1.1$ & 1298.717 & $5.2 \pm 1.0$ \\
\hline 1299.217 & $5.1 \pm 0.9$ & 1299.717 & $5.4 \pm 1.1$ & 1300.217 & $5.2 \pm 1.0$ & 1300.717 & $5.1 \pm 0.9$ \\
\hline 1301.217 & $5.3 \pm 1.0$ & 1301.717 & $5.2 \pm 0.9$ & 1302.217 & $5.2 \pm 1.0$ & 1302.717 & $5.2 \pm 0.9$ \\
\hline 1303.217 & $5.2 \pm 0.9$ & 1303.717 & $5.3 \pm 1.0$ & 1304.217 & $5.3 \pm 1.0$ & 1304.717 & $5.2 \pm 0.9$ \\
\hline 1305.217 & $5.2 \pm 0.9$ & 1305.717 & $5.2 \pm 0.9$ & 1306.217 & $5.2 \pm 0.9$ & 1306.717 & $5.3 \pm 1.0$ \\
\hline
\end{tabular}


TABLE II. (Continued)

\begin{tabular}{|c|c|c|c|c|c|c|c|}
\hline$f($ in $\mathrm{Hz})$ & $h_{0}^{90 \%} \times 10^{24}$ & $f($ in $\mathrm{Hz})$ & $h_{0}^{90 \%} \times 10^{24}$ & $f($ in $\mathrm{Hz})$ & $h_{0}^{90 \%} \times 10^{24}$ & $f($ in $\mathrm{Hz})$ & $h_{0}^{90 \%} \times 10^{24}$ \\
\hline 1307.217 & $5.3 \pm 1.0$ & 1307.717 & $5.5 \pm 1.1$ & 1308.217 & $5.2 \pm 0.9$ & 1308.717 & $5.4 \pm 1.1$ \\
\hline 1309.217 & $5.2 \pm 0.9$ & 1309.717 & $5.3 \pm 1.0$ & 1310.217 & $5.5 \pm 1.1$ & 1310.717 & $5.4 \pm 1.0$ \\
\hline 1311.217 & $5.2 \pm 1.0$ & 1311.717 & $5.3 \pm 1.0$ & 1312.217 & $5.2 \pm 0.9$ & 1312.717 & $5.3 \pm 1.0$ \\
\hline 1313.217 & $5.4 \pm 1.0$ & 1313.717 & $5.2 \pm 0.9$ & 1314.217 & $5.2 \pm 0.9$ & 1314.717 & $5.4 \pm 1.0$ \\
\hline 1315.217 & $5.5 \pm 1.1$ & 1315.717 & $5.5 \pm 1.1$ & 1316.217 & $5.2 \pm 0.9$ & 1316.717 & $5.2 \pm 0.9$ \\
\hline 1317.217 & $5.4 \pm 1.0$ & 1317.717 & $5.5 \pm 1.1$ & 1318.217 & $5.2 \pm 0.9$ & 1318.717 & $5.3 \pm 0.9$ \\
\hline 1320.717 & $5.3 \pm 0.9$ & 1321.217 & $5.3 \pm 0.9$ & 1321.717 & $5.2 \pm 0.9$ & 1322.217 & $5.4 \pm 1.0$ \\
\hline 1322.717 & $5.2 \pm 0.9$ & 1323.217 & $5.2 \pm 0.9$ & 1323.717 & $5.3 \pm 1.0$ & 1324.217 & $5.4 \pm 1.0$ \\
\hline 1324.717 & $5.2 \pm 0.9$ & 1325.217 & $5.5 \pm 1.1$ & 1325.717 & $5.4 \pm 1.0$ & 1326.217 & $5.2 \pm 0.9$ \\
\hline 1326.717 & $5.5 \pm 1.1$ & 1327.217 & $5.2 \pm 0.9$ & 1327.717 & $5.2 \pm 0.9$ & 1328.217 & $5.2 \pm 0.9$ \\
\hline 1328.717 & $5.4 \pm 1.0$ & 1329.217 & $5.2 \pm 0.9$ & 1329.717 & $5.4 \pm 1.0$ & 1330.217 & $5.2 \pm 0.9$ \\
\hline 1330.717 & $5.4 \pm 1.0$ & 1331.217 & $5.3 \pm 1.0$ & 1331.717 & $5.2 \pm 0.9$ & 1332.217 & $5.2 \pm 0.9$ \\
\hline 1332.717 & $5.2 \pm 0.9$ & 1333.217 & $5.2 \pm 0.9$ & 1333.717 & $5.2 \pm 0.9$ & 1334.217 & $5.4 \pm 1.0$ \\
\hline 1334.717 & $5.2 \pm 0.9$ & 1335.217 & $5.2 \pm 0.9$ & 1335.717 & $5.4 \pm 1.0$ & 1336.217 & $5.3 \pm 0.9$ \\
\hline 1336.717 & $5.3 \pm 1.0$ & 1337.217 & $5.3 \pm 1.0$ & 1337.717 & $5.3 \pm 0.9$ & 1338.217 & $5.6 \pm 1.1$ \\
\hline 1338.717 & $5.3 \pm 0.9$ & 1339.217 & $5.3 \pm 1.0$ & 1339.717 & $5.4 \pm 1.0$ & 1340.217 & $5.3 \pm 0.9$ \\
\hline 1340.717 & $5.4 \pm 1.0$ & 1341.217 & $5.3 \pm 0.9$ & 1341.717 & $5.3 \pm 0.9$ & 1342.217 & $5.3 \pm 0.9$ \\
\hline 1342.717 & $5.4 \pm 1.0$ & 1343.217 & $5.6 \pm 1.1$ & 1343.717 & $5.3 \pm 0.9$ & 1344.217 & $5.3 \pm 0.9$ \\
\hline 1344.717 & $5.4 \pm 1.0$ & 1345.217 & $5.4 \pm 1.0$ & 1345.717 & $5.3 \pm 0.9$ & 1346.217 & $5.3 \pm 0.9$ \\
\hline 1346.717 & $5.3 \pm 0.9$ & 1347.217 & $5.3 \pm 0.9$ & 1347.717 & $5.6 \pm 1.2$ & 1348.217 & $5.3 \pm 0.9$ \\
\hline 1348.717 & $5.3 \pm 0.9$ & 1349.217 & $5.4 \pm 1.0$ & 1349.717 & $5.4 \pm 1.0$ & 1350.217 & $5.3 \pm 0.9$ \\
\hline 1350.717 & $5.3 \pm 0.9$ & 1351.217 & $5.4 \pm 1.0$ & 1351.717 & $5.4 \pm 1.0$ & 1352.217 & $5.3 \pm 0.9$ \\
\hline 1352.717 & $5.3 \pm 0.9$ & 1353.217 & $5.3 \pm 0.9$ & 1353.717 & $5.3 \pm 0.9$ & 1354.217 & $5.3 \pm 0.9$ \\
\hline 1354.717 & $5.6 \pm 1.2$ & 1355.217 & $5.4 \pm 1.0$ & 1355.717 & $5.3 \pm 0.9$ & 1356.217 & $5.3 \pm 0.9$ \\
\hline 1356.717 & $5.5 \pm 1.1$ & 1357.217 & $5.6 \pm 1.1$ & 1357.717 & $5.3 \pm 0.9$ & 1358.217 & $5.3 \pm 0.9$ \\
\hline 1358.717 & $5.3 \pm 0.9$ & 1359.217 & $5.3 \pm 0.9$ & 1359.717 & $5.5 \pm 1.1$ & 1360.217 & $5.3 \pm 0.9$ \\
\hline 1360.717 & $5.4 \pm 1.0$ & 1361.217 & $5.5 \pm 1.1$ & 1361.717 & $5.3 \pm 0.9$ & 1362.217 & $5.3 \pm 0.9$ \\
\hline 1362.717 & $5.7 \pm 1.2$ & 1363.217 & $5.4 \pm 0.9$ & 1363.717 & $5.4 \pm 0.9$ & 1364.217 & $5.4 \pm 0.9$ \\
\hline 1364.717 & $5.5 \pm 1.1$ & 1365.217 & $5.4 \pm 0.9$ & 1365.717 & $5.4 \pm 0.9$ & 1366.217 & $5.4 \pm 1.1$ \\
\hline 1366.717 & $5.4 \pm 0.9$ & 1367.217 & $5.4 \pm 0.9$ & 1367.717 & $5.5 \pm 1.1$ & 1368.217 & $5.5 \pm 1.1$ \\
\hline 1368.717 & $5.5 \pm 1.1$ & 1369.217 & $5.7 \pm 1.1$ & 1369.717 & $5.4 \pm 0.9$ & 1370.217 & $5.5 \pm 1.1$ \\
\hline 1370.717 & $5.4 \pm 0.9$ & 1371.217 & $5.4 \pm 0.9$ & 1371.717 & $5.5 \pm 1.1$ & 1372.217 & $5.4 \pm 0.9$ \\
\hline 1372.717 & $5.9 \pm 1.1$ & 1373.217 & $5.7 \pm 1.2$ & 1373.717 & $5.5 \pm 1.0$ & 1374.217 & $6.1 \pm 1.2$ \\
\hline 1374.717 & $5.7 \pm 1.1$ & 1375.217 & $5.5 \pm 1.0$ & 1375.717 & $5.5 \pm 1.1$ & 1376.217 & $5.4 \pm 0.9$ \\
\hline 1376.717 & $5.7 \pm 1.1$ & 1377.217 & $5.5 \pm 1.0$ & 1377.717 & $5.6 \pm 1.1$ & 1378.217 & $5.7 \pm 1.0$ \\
\hline 1378.717 & $5.5 \pm 1.0$ & 1380.717 & $5.5 \pm 1.1$ & 1381.217 & $5.4 \pm 0.9$ & 1381.717 & $5.7 \pm 1.2$ \\
\hline 1382.217 & $5.4 \pm 0.9$ & 1382.717 & $5.5 \pm 1.1$ & 1383.217 & $5.4 \pm 0.9$ & 1383.717 & $5.5 \pm 1.1$ \\
\hline 1384.217 & $5.4 \pm 0.9$ & 1384.717 & $5.4 \pm 0.9$ & 1385.217 & $5.5 \pm 1.1$ & 1385.717 & $5.4 \pm 0.9$ \\
\hline 1386.217 & $5.6 \pm 1.1$ & 1386.717 & $5.7 \pm 1.1$ & 1387.217 & $5.6 \pm 1.0$ & 1387.717 & $5.5 \pm 1.0$ \\
\hline 1388.717 & $5.7 \pm 1.1$ & 1389.217 & $5.8 \pm 1.2$ & 1389.717 & $5.5 \pm 1.0$ & 1390.217 & $5.6 \pm 1.1$ \\
\hline 1390.717 & $5.9 \pm 1.2$ & 1391.217 & $6.1 \pm 1.1$ & 1391.717 & $5.6 \pm 1.0$ & 1392.217 & $5.8 \pm 1.2$ \\
\hline 1392.717 & $5.6 \pm 1.1$ & 1393.217 & $5.8 \pm 1.2$ & 1393.717 & $5.5 \pm 1.0$ & 1394.217 & $5.6 \pm 1.1$ \\
\hline 1394.717 & $5.5 \pm 1.0$ & 1395.217 & $5.5 \pm 1.0$ & 1395.717 & $5.5 \pm 1.0$ & 1396.217 & $5.5 \pm 1.0$ \\
\hline 1396.717 & $5.5 \pm 1.0$ & 1397.217 & $5.6 \pm 1.1$ & 1397.717 & $5.6 \pm 1.1$ & 1398.217 & $5.6 \pm 1.1$ \\
\hline 1398.717 & $5.5 \pm 1.0$ & 1399.217 & $5.5 \pm 1.0$ & 1399.717 & $5.8 \pm 1.2$ & 1400.717 & $5.8 \pm 1.2$ \\
\hline 1401.217 & $5.8 \pm 1.2$ & 1401.717 & $5.8 \pm 1.1$ & 1402.217 & $5.5 \pm 1.0$ & 1402.717 & $5.5 \pm 1.0$ \\
\hline 1403.217 & $5.5 \pm 1.0$ & 1403.717 & $5.5 \pm 1.0$ & 1404.217 & $5.5 \pm 1.0$ & 1404.717 & $5.8 \pm 1.2$ \\
\hline 1405.217 & $5.7 \pm 1.1$ & 1405.717 & $5.5 \pm 1.0$ & 1406.217 & $5.5 \pm 1.0$ & 1406.717 & $5.6 \pm 1.1$ \\
\hline 1407.217 & $5.5 \pm 1.0$ & 1407.717 & $5.7 \pm 1.1$ & 1408.217 & $5.5 \pm 1.0$ & 1408.717 & $5.6 \pm 1.1$ \\
\hline 1409.217 & $5.5 \pm 1.0$ & 1409.717 & $5.5 \pm 1.0$ & 1410.217 & $5.5 \pm 1.0$ & 1410.717 & $5.5 \pm 1.0$ \\
\hline 1411.217 & $5.5 \pm 1.0$ & 1411.717 & $5.6 \pm 1.1$ & 1412.217 & $5.5 \pm 1.0$ & 1412.717 & $5.6 \pm 1.1$ \\
\hline 1413.217 & $5.5 \pm 1.0$ & 1413.717 & $5.5 \pm 1.0$ & 1414.217 & $5.5 \pm 1.0$ & 1414.717 & $5.6 \pm 1.1$ \\
\hline 1415.217 & $5.5 \pm 1.0$ & 1415.717 & $5.6 \pm 1.0$ & 1416.217 & $5.7 \pm 1.1$ & 1416.717 & $5.6 \pm 1.1$ \\
\hline 1417.217 & $5.7 \pm 1.1$ & 1417.717 & $5.6 \pm 1.0$ & 1418.217 & $5.6 \pm 1.0$ & 1418.717 & $5.7 \pm 1.1$ \\
\hline 1419.217 & $5.6 \pm 1.0$ & 1419.717 & $5.6 \pm 1.0$ & 1420.217 & $5.6 \pm 1.0$ & 1420.717 & $5.6 \pm 1.0$ \\
\hline
\end{tabular}


TABLE II. (Continued)

\begin{tabular}{|c|c|c|c|c|c|c|c|}
\hline$f($ in $\mathrm{Hz})$ & $h_{0}^{90 \%} \times 10^{24}$ & $f($ in $\mathrm{Hz})$ & $h_{0}^{90 \%} \times 10^{24}$ & $f($ in $\mathrm{Hz})$ & $h_{0}^{90 \%} \times 10^{24}$ & $f($ in $\mathrm{Hz})$ & $h_{0}^{90 \%} \times 10^{24}$ \\
\hline 1421.217 & $5.8 \pm 1.1$ & 1421.717 & $5.6 \pm 1.0$ & 1422.217 & $5.6 \pm 1.0$ & 1422.717 & $5.6 \pm 1.0$ \\
\hline 1423.217 & $5.6 \pm 1.0$ & 1423.717 & $5.6 \pm 1.0$ & 1424.217 & $5.7 \pm 1.1$ & 1424.717 & $5.6 \pm 1.0$ \\
\hline 1425.217 & $5.6 \pm 1.0$ & 1425.717 & $5.6 \pm 1.0$ & 1426.217 & $5.7 \pm 1.1$ & 1426.717 & $5.8 \pm 1.1$ \\
\hline 1427.217 & $5.8 \pm 1.1$ & 1427.717 & $5.6 \pm 1.0$ & 1428.217 & $5.8 \pm 1.1$ & 1428.717 & $5.8 \pm 1.1$ \\
\hline 1429.217 & $5.8 \pm 1.1$ & 1429.717 & $5.8 \pm 1.1$ & 1430.217 & $5.6 \pm 1.0$ & 1430.717 & $5.6 \pm 1.0$ \\
\hline 1431.217 & $5.6 \pm 1.0$ & 1431.717 & $5.6 \pm 1.0$ & 1432.217 & $5.6 \pm 1.0$ & 1432.717 & $5.6 \pm 1.0$ \\
\hline 1433.217 & $5.6 \pm 1.0$ & 1433.717 & $6.0 \pm 1.2$ & 1434.217 & $5.8 \pm 1.1$ & 1434.717 & $5.8 \pm 1.1$ \\
\hline 1435.217 & $5.6 \pm 1.0$ & 1435.717 & $5.6 \pm 1.0$ & 1436.217 & $5.8 \pm 1.1$ & 1436.717 & $5.8 \pm 1.1$ \\
\hline 1437.217 & $5.6 \pm 1.0$ & 1437.717 & $5.8 \pm 1.1$ & 1438.217 & $5.9 \pm 1.1$ & 1438.717 & $5.8 \pm 1.1$ \\
\hline 1440.717 & $5.7 \pm 1.0$ & 1441.217 & $5.7 \pm 1.0$ & 1441.717 & $5.7 \pm 1.0$ & 1442.217 & $5.7 \pm 1.1$ \\
\hline 1442.717 & $5.9 \pm 1.2$ & 1443.217 & $5.7 \pm 1.0$ & 1443.717 & $5.7 \pm 1.0$ & 1444.217 & $5.7 \pm 1.1$ \\
\hline 1444.717 & $5.7 \pm 1.0$ & 1445.217 & $5.7 \pm 1.0$ & 1445.717 & $5.7 \pm 1.0$ & 1446.217 & $5.7 \pm 1.0$ \\
\hline 1446.717 & $5.8 \pm 1.1$ & 1447.217 & $5.7 \pm 1.0$ & 1447.717 & $5.7 \pm 1.0$ & 1448.217 & $5.7 \pm 1.0$ \\
\hline 1448.717 & $5.9 \pm 1.1$ & 1449.217 & $5.8 \pm 1.1$ & 1449.717 & $5.7 \pm 1.0$ & 1450.217 & $5.7 \pm 1.0$ \\
\hline 1450.717 & $5.8 \pm 1.1$ & 1451.217 & $5.7 \pm 1.0$ & 1451.717 & $6.0 \pm 1.2$ & 1452.217 & $5.7 \pm 1.0$ \\
\hline 1452.717 & $5.7 \pm 1.0$ & 1453.217 & $5.9 \pm 1.1$ & 1453.717 & $5.8 \pm 1.1$ & 1454.217 & $5.8 \pm 1.1$ \\
\hline 1454.717 & $5.7 \pm 1.0$ & 1455.217 & $5.8 \pm 1.1$ & 1455.717 & $5.7 \pm 1.0$ & 1456.217 & $6.0 \pm 1.2$ \\
\hline 1456.717 & $5.9 \pm 1.1$ & 1457.217 & $5.7 \pm 1.0$ & 1457.717 & $5.7 \pm 1.0$ & 1458.217 & $6.0 \pm 1.2$ \\
\hline 1458.717 & $5.7 \pm 1.0$ & 1459.217 & $5.8 \pm 1.1$ & 1459.717 & $5.7 \pm 1.0$ & 1460.217 & $5.8 \pm 1.1$ \\
\hline 1460.717 & $5.7 \pm 1.0$ & 1461.217 & $5.7 \pm 1.0$ & 1461.717 & $5.7 \pm 1.0$ & 1462.217 & $5.7 \pm 1.0$ \\
\hline 1462.717 & $5.7 \pm 1.0$ & 1463.217 & $5.7 \pm 1.0$ & 1463.717 & $5.7 \pm 1.0$ & 1464.217 & $5.7 \pm 1.0$ \\
\hline 1464.717 & $5.7 \pm 1.0$ & 1465.217 & $5.8 \pm 1.1$ & 1465.717 & $5.9 \pm 1.1$ & 1466.217 & $5.7 \pm 1.0$ \\
\hline 1466.717 & $5.8 \pm 1.1$ & 1467.217 & $5.7 \pm 1.0$ & 1467.717 & $5.8 \pm 1.1$ & 1468.217 & $5.8 \pm 1.1$ \\
\hline 1468.717 & $5.9 \pm 1.1$ & 1469.217 & $5.8 \pm 1.0$ & 1469.717 & $5.8 \pm 1.0$ & 1470.217 & $6.0 \pm 1.2$ \\
\hline 1470.717 & $5.8 \pm 1.0$ & 1471.217 & $5.8 \pm 1.0$ & 1471.717 & $5.8 \pm 1.0$ & 1472.217 & $5.8 \pm 1.0$ \\
\hline 1472.717 & $5.8 \pm 1.0$ & 1473.217 & $6.1 \pm 1.3$ & 1473.717 & $5.9 \pm 1.1$ & 1474.217 & $5.9 \pm 1.1$ \\
\hline 1474.717 & $6.1 \pm 1.2$ & 1475.217 & $5.8 \pm 1.0$ & 1475.717 & $6.1 \pm 1.3$ & 1476.217 & $6.0 \pm 1.2$ \\
\hline 1476.717 & $5.8 \pm 1.0$ & 1477.217 & $5.8 \pm 1.0$ & 1477.717 & $6.1 \pm 1.2$ & 1478.217 & $5.9 \pm 1.1$ \\
\hline 1478.717 & $5.8 \pm 1.0$ & 1479.217 & $5.9 \pm 1.1$ & 1479.717 & $6.0 \pm 1.2$ & 1480.217 & $5.8 \pm 1.0$ \\
\hline 1480.717 & $5.9 \pm 1.1$ & 1481.217 & $5.8 \pm 1.0$ & 1481.717 & $5.8 \pm 1.0$ & 1482.217 & $5.8 \pm 1.0$ \\
\hline 1482.717 & $5.8 \pm 1.0$ & 1483.217 & $5.8 \pm 1.0$ & 1483.717 & $5.8 \pm 1.0$ & 1484.217 & $5.9 \pm 1.1$ \\
\hline 1484.717 & $5.8 \pm 1.0$ & 1485.217 & $6.1 \pm 1.2$ & 1485.717 & $6.0 \pm 1.2$ & 1486.217 & $5.8 \pm 1.0$ \\
\hline 1486.717 & $5.9 \pm 1.1$ & 1487.217 & $5.8 \pm 1.0$ & 1487.717 & $5.9 \pm 1.1$ & 1488.217 & $5.8 \pm 1.0$ \\
\hline 1488.717 & $5.8 \pm 1.0$ & 1489.217 & $6.1 \pm 1.2$ & 1489.717 & $5.8 \pm 1.0$ & 1490.217 & $5.9 \pm 1.1$ \\
\hline 1490.717 & $5.8 \pm 1.0$ & 1491.217 & $5.8 \pm 1.0$ & 1491.717 & $5.8 \pm 1.0$ & 1492.217 & $5.8 \pm 1.0$ \\
\hline 1492.717 & $5.8 \pm 1.0$ & 1493.217 & $5.8 \pm 1.0$ & 1493.717 & $5.9 \pm 1.1$ & 1494.217 & $5.8 \pm 1.0$ \\
\hline 1494.717 & $5.9 \pm 1.1$ & 1495.217 & $5.8 \pm 1.0$ & 1495.717 & $6.1 \pm 1.2$ & 1496.217 & $5.9 \pm 1.0$ \\
\hline 1496.717 & $5.9 \pm 1.0$ & 1497.217 & $5.9 \pm 1.0$ & 1497.717 & $6.0 \pm 1.2$ & 1498.217 & $6.0 \pm 1.2$ \\
\hline 1498.717 & $6.2 \pm 1.3$ & - & - & - & - & - & - \\
\hline
\end{tabular}




\section{Detector lines}

See Table III.

TABLE III. Instrumental lines identified and cleaned before the Einstein@Home analysis. The different columns represent: (I) the source of the line; (II) the central frequency of the instrumental line; (III) the number of harmonics in the signal-frequency range, i.e. between 1249.7 and $1499.7 \mathrm{~Hz}$; (IV) the low-frequency side (LFS) of the knockout band; (V) the high-frequency side (HFS) of the knockout band; (VI) the interferometer where the instrumental lines were identified. Note that when there are higher harmonics present, the knockout bandwidth remains constant.

\begin{tabular}{llcccc}
\hline \hline Source & $f(\mathrm{~Hz})$ & Harmonics & LFS $(\mathrm{Hz})$ & HFS $(\mathrm{Hz})$ & IFO \\
\hline Power mains & 60.0 & 5 & 1.0 & 1.0 & $\mathrm{~L}, \mathrm{H}$ \\
Violin mode & 1373.75 & 1 & 0.1 & 0.1 & $\mathrm{H}$ \\
Violin mode & 1374.44 & 1 & 0.1 & 0.1 & $\mathrm{H}$ \\
Violin mode & 1377.14 & 1 & 0.1 & 0.1 & $\mathrm{H}$ \\
Violin mode & 1378.75 & 1 & 0.1 & 0.1 & $\mathrm{H}$ \\
Violin mode & 1379.52 & 1 & 0.1 & 0.06 & $\mathrm{H}$ \\
Violin mode & 1389.06 & 1 & 0.06 & 0.07 & $\mathrm{H}$ \\
Violin mode & 1389.82 & 1 & 0.07 & 0.2 & $\mathrm{H}$ \\
Violin mode & 1391.5 & 1 & 0.2 & 0.075 & $\mathrm{H}$ \\
Violin mode & 1372.925 & 1 & 0.075 & 0.1 & $\mathrm{~L}$ \\
Violin mode & 1374.7 & 1 & 0.1 & 0.1 & $\mathrm{~L}$ \\
Violin mode & 1375.2 & 1 & 0.1 & 0.1 & $\mathrm{~L}$ \\
Violin mode & 1378.39 & 1 & 0.05 & 0.05 & $\mathrm{~L}$ \\
Violin mode & 1387.4 & 1 & 0.3 & 0.3 & $\mathrm{~L}$ \\
Violin mode & 1388.5 & 1 & &
\end{tabular}

\section{Signal-frequency ranges and data quality}

See Table IV.

TABLE IV. Signal-frequency ranges where the results might have contributions from fake data. When the results are entirely due to artificial data, the band is listed in the "All fake data" column; bands where the results comprised of contributions from both fake and real data are listed in the other three columns. The "Mixed (left)" and "Mixed (right)" columns are populated only when there is a matching "All fake data" entry, which highlights the same physical cause for the fake data, i.e. the cleaning. The "Mixed (isolated)" column lists isolated ranges of mixed data. The list of input data frequencies where the data was substituted with artificial noise are given in Table III.

\begin{tabular}{|c|c|c|c|c|c|}
\hline Source & Mixed (isolated) & Mixed (left) & All fake data & Mixed (right) & IFO \\
\hline Power mains & $\ldots$ & $1258.7976-1259.2024$ & $1259.2024-1260.7974$ & $1260.7974-1261.2026$ & $\mathrm{H}, \mathrm{L}$ \\
\hline Power mains & $\cdots$ & $1318.7915-1319.2085$ & $1319.2085-1320.7913$ & $1320.7913-1321.2087$ & $\mathrm{H}, \mathrm{L}$ \\
\hline Violin mode & $1372.6360-1373.2140$ & $\cdots$ & $\cdots$ & $\ldots$ & $\mathrm{L}$ \\
\hline Violin mode & $1373.4359-1374.0641$ & $\ldots$ & $\ldots$ & $\ldots$ & $\mathrm{H}$ \\
\hline Violin mode & $1374.1259-1375.5142$ & $\ldots$ & $\ldots$ & $\ldots$ & $\mathrm{H}, \mathrm{L}$ \\
\hline Violin mode & $1376.8256-1377.4554$ & $\ldots$ & $\ldots$ & $\ldots$ & $\mathrm{H}$ \\
\hline Violin mode & $1378.0755-1379.0646$ & $\ldots$ & $\ldots$ & $\ldots$ & $\mathrm{H}, \mathrm{L}$ \\
\hline Violin mode & $1379.2054-1379.8347$ & $\cdots$ & $\cdots$ & $\ldots$ & $\mathrm{H}$ \\
\hline Power mains & $\ldots$ & $1378.7854-1379.2146$ & $1379.2146-1380.7852$ & $1380.7852-1381.2148$ & $\mathrm{H}, \mathrm{L}$ \\
\hline Violin mode & $1387.1346-1387.6655$ & $\ldots$ & $\ldots$ & $\ldots$ & $\mathrm{L}$ \\
\hline Violin mode & $\ldots$ & $1387.9845-1388.4155$ & $1388.4155-1388.5844$ & $1388.5844-1389.0156$ & $\mathrm{H}, \mathrm{L}$ \\
\hline Violin mode & $1388.7844-1389.3356$ & $\ldots$ & $\ldots$ & $\ldots$ & $\mathrm{H}, \mathrm{L}$ \\
\hline Violin mode & $1389.5343-1390.1057$ & $\ldots$ & $\ldots$ & $\cdots$ & $\mathrm{H}$ \\
\hline Violin mode & $1391.0842-1391.9159$ & $\ldots$ & $\ldots$ & $\ldots$ & $\mathrm{H}, \mathrm{L}$ \\
\hline Power mains & .. & $1438.7793-1439.2207$ & $1439.2207-1440.7791$ & $1440.7791-1441.2209$ & $\mathrm{H}, \mathrm{L}$ \\
\hline Power mains & $\ldots$ & $1498.7732-1499.2268$ & $1499.2268-1499.7170$ & $\ldots$ & $\mathrm{H}, \mathrm{L}$ \\
\hline
\end{tabular}




\section{Omitted $50 \mathrm{mHz}$ bands from signal frequency}

See Table V.

TABLE V. $50 \mathrm{mHz}$ search-frequency bands that were identified as "disturbed" based on visual inspection (type D), or where the results were produced from "All fake data," as detailed in Table IV (type C). Both sets of bands (type D and C) were excluded from the analysis. The first two columns list the starting frequency of the first and last $50 \mathrm{mHz}$ band in the contiguous range of excluded bands.

\begin{tabular}{|c|c|c|c|c|c|}
\hline$f_{\text {start }}($ in $\mathrm{Hz})$ & $f_{\text {end }}($ in $\mathrm{Hz})$ & Type & $f_{\text {start }}($ in $\mathrm{Hz})$ & $f_{\text {end }}($ in $\mathrm{Hz})$ & Type \\
\hline 1258.617 & 1258.717 & $\mathrm{D}$ & 1259.217 & 1260.717 & $\mathrm{C}$ \\
\hline 1291.017 & 1291.067 & $\mathrm{D}$ & 1292.567 & 1292.867 & $\mathrm{D}$ \\
\hline 1293.267 & 1293.567 & $\mathrm{D}$ & 1293.917 & 1294.217 & D \\
\hline 1296.367 & 1296.817 & $\mathrm{D}$ & 1297.517 & 1297.717 & D \\
\hline 1298.667 & 1298.967 & $\mathrm{D}$ & 1313.467 & 1313.517 & D \\
\hline 1318.567 & 1318.667 & $\mathrm{D}$ & 1319.217 & 1320.717 & $\mathrm{C}$ \\
\hline 1372.867 & 1373.167 & $\mathrm{D}$ & 1376.417 & 1376.817 & $\mathrm{D}$ \\
\hline 1378.517 & 1378.617 & $\mathrm{D}$ & 1379.217 & 1380.717 & $\mathrm{C}$ \\
\hline 1382.567 & $\ldots$ & $\mathrm{D}$ & 1387.317 & $\ldots$ & $\mathrm{D}$ \\
\hline 1387.767 & 1388.217 & $\mathrm{D}$ & 1388.417 & 1388.517 & $\mathrm{C}$ \\
\hline 1389.467 & $\ldots$ & $\mathrm{D}$ & 1389.767 & 1390.217 & $\mathrm{D}$ \\
\hline 1390.467 & 1390.867 & $\mathrm{D}$ & 1390.967 & 1391.117 & D \\
\hline 1395.217 & 1395.467 & $\mathrm{D}$ & 1398.417 & 1398.667 & $\mathrm{D}$ \\
\hline 1399.967 & 1400.867 & $\mathrm{D}$ & 1400.967 & 1401.267 & $\mathrm{D}$ \\
\hline 1438.417 & 1438.517 & $\mathrm{D}$ & 1439.267 & 1440.717 & $\mathrm{C}$ \\
\hline 1453.467 & 1453.517 & $\mathrm{D}$ & 1454.967 & 1455.067 & $\mathrm{D}$ \\
\hline 1498.317 & 1498.467 & $\mathrm{D}$ & 1499.267 & 1499.667 & $\mathrm{C}$ \\
\hline
\end{tabular}

[1] J. Aasi et al. (LIGO Scientific Collaboration), Einstein@ Home all-sky search for periodic gravitational waves in LIGO S5 data, Phys. Rev. D 87, 042001 (2013).

[2] B. Abbot et al. (LIGO Scientific Collaboration), Searches for periodic gravitational waves from unknown isolated sources and Scorpius X-1: Results from the second LIGO science run, Phys. Rev. D 76, 082001 (2007).

[3] B. Abbot et al. (LIGO Scientific Collaboration), All-sky search for periodic gravitational waves in LIGO S4 data, Phys. Rev. D 77, 022001 (2008).

[4] B. Abbot et al. (LIGO Scientific Collaboration), All-Sky LIGO Search for Periodic Gravitational Waves in the Early Fifth-Science-Run Data, Phys. Rev. Lett. 102, 111102 (2009).

[5] B. Abbot et al. (LIGO Scientific Collaboration), All-sky search for periodic gravitational waves in the full S5 LIGO data, Phys. Rev. D 85, 022001 (2012).

[6] B. Abbot et al. (LIGO Scientific Collaboration), Results of the deepest all-sky survey for continuous gravitational waves on LIGO S6 data running on the Einstein@Home volunteer distributed computing project, arXiv:1606.09619.

[7] B. Abbot et al. (LIGO Scientific Collaboration), Comprehensive all-sky search for periodic gravitational waves in the sixth science run LIGO data, arXiv:1605.03233.
[8] H. J. Pletsch, Parameter-space correlations of the optimal statistic for continuous gravitational-wave detection, Phys. Rev. D 78, 102005 (2008).

[9] H. J. Pletsch, Parameter-space metric of semicoherent searches for continuous gravitational waves, Phys. Rev. D 82, 042002 (2010).

[10] J. Abadie et al. (LIGO Scientific Collaboration), Calibration of the LIGO gravitational wave detectors in the fifth science run, Nucl. Instrum. Methods Phys. Res., Sect. A 624, 223 (2010).

[11] B. Abbot et al. (LIGO Scientific Collaboration), LIGO: The laser interferometer gravitational-wave observatory, Rep. Prog. Phys. 72, 076901 (2009).

[12] C. Cutler and B. F. Schutz, Generalized $\mathcal{F}$-statistic: Multiple detectors and multiple gravitational wave pulsars, Phys. Rev. D 72, 063006 (2005).

[13] M. A. Papa et al., Hierarchical follow-up of sub-threshold candidates of an all-sky Einstein@home search for continuous gravitational waves on LIGO data (to be published).

[14] J. Aasi et al. (LIGO Scientific Collaboration), Directed search for continuous gravitational waves from the Galactic center, Phys. Rev. D 88, 102002 (2013).

[15] K. Wette, Estimating the sensitivity of wide-parameterspace searches for gravitational-wave pulsars, Phys. Rev. D 85, 042003 (2012). 
[16] J. Ming, B. Krishnan, M. A. Papa, C. Aulbert, and H. Fehrmann, Optimal directed searches for continuous gravitational waves, Phys. Rev. D 93, 064011 (2016).

[17] N. K. Johnson-McDaniel and B. J. Owen, Maximum elastic deformations of relativistic stars, Phys. Rev. D 88, 044004 (2013).

[18] D. Keitel, Robust semicoherent searches for continuous gravitational waves with noise and signal models including hours to days long transients, Phys. Rev. D 93, 084024 (2016).

[19] R. Prix, S. Giampanis, and C. Messenger, Search method for long-duration gravitational-wave transients from neutron stars, Phys. Rev. D 84, 023007 (2011).

[20] A. Singh, Gravitational Wave transient signal emission via Ekman Pumping in Neutron Stars during post-glitch relaxation phase, arXiv:1605.08420. 